Bull. Korean Math. Soc. 51 (2014), No. 6, pp. 1615-1623

http://dx.doi.org/10.4134/BKMS.2014.51.6.1615

\title{
GENERALIZED MATRIX FUNCTIONS, IRREDUCIBILITY AND EQUALITY
}

\author{
Mohammad Hossein Jafari and Ali Reza Madadi
}

\begin{abstract}
Let $G \leq S_{n}$ and $\chi$ be any nonzero complex valued function on $G$. We first study the irreducibility of the generalized matrix polynomial $d_{\chi}^{G}(X)$, where $X=\left(x_{i j}\right)$ is an $n$-by- $n$ matrix whose entries are $n^{2}$ commuting independent indeterminates over $\mathbb{C}$. In particular, we show that if $\chi$ is an irreducible character of $G$, then $d_{\chi}^{G}(X)$ is an irreducible polynomial, where either $G=S_{n}$ or $G=A_{n}$ and $n \neq 2$. We then give a necessary and sufficient condition for the equality of two generalized matrix functions on the set of the so-called $\chi$-singular ( $\chi$-nonsingular) matrices.
\end{abstract}

\section{Introduction}

Let $S_{n}$ be the symmetric group of degree $n, G$ an arbitrary subgroup of $S_{n}$, and let $\chi: G \rightarrow \mathbb{C}$ be a function. Denote by $M_{n}(\mathbb{C})$ the set of all $n$-by- $n$ matrices over $\mathbb{C}$ and define the function $d_{\chi}^{G}: M_{n}(\mathbb{C}) \rightarrow \mathbb{C}$ as follows:

$$
d_{\chi}^{G}(A)=\sum_{\sigma \in G} \chi(\sigma) \prod_{i=1}^{n} a_{i \sigma(i)},
$$

where $A=\left(a_{i j}\right) \in M_{n}(\mathbb{C})$. The function $d_{\chi}^{G}$ is called the generalized matrix function associated with $G$ and $\chi$. Note that if $G=S_{n}$ and $\chi=1_{G}$ is the principal character of $G$, then $d_{\chi}^{G}=$ per is the permanent, and if $G=S_{n}$ and $\chi=\varepsilon$ is the alternating character of $G$, then $d_{\chi}^{G}=\operatorname{det}$ is the determinant. We refer the reader to [4] and [5] for more information about generalized matrix functions. Now let $X=\left(x_{i j}\right)$ be an $n$-by- $n$ matrix whose entries are $n^{2}$ commuting independent indeterminates over $\mathbb{C}$. Therefore,

$$
d_{\chi}^{G}(X)=\sum_{\sigma \in G} \chi(\sigma) \prod_{i=1}^{n} x_{i \sigma(i)}
$$

Received August 14, 2013.

2010 Mathematics Subject Classification. 15A15, 12E05, $20 \mathrm{C} 15$.

Key words and phrases. generalized matrix functions, irreducibility, $\chi$-singular and $\chi$ nonsingular matrices. 
can be viewed as an element of $\mathbb{C}\left[x_{11}, x_{12}, \ldots, x_{n n}\right]$, the polynomial ring in the variables $x_{11}, x_{12}, \ldots, x_{n n}$ with coefficients in $\mathbb{C}$. We call $d_{\chi}^{G}(X)$ the generalized matrix polynomial associated with $G$ and $\chi$.

Our main aim in this paper is as follows: in Section 2, we first prove some results about the irreducibility of polynomials and then, using them, we obtain the irreducibility of $d_{\chi}^{G}(X)$ under some restrictions over $G$ and $\chi$; in Section 3, by using Hilbert's Nullstellensatz and results of Section 2, we give a criterion for the equality of two generalized matrix functions on the set of the so-called $\chi$-singular $(\chi$-nonsingular) matrices.

\section{Irreducibility of $d_{\chi}^{G}(X)$}

First we recall a few standard notation and definitions. Let $\mathbb{C}\left[x_{1}, x_{2}, \ldots, x_{n}\right]$ be the polynomial ring in the variables $x_{1}, x_{2}, \ldots, x_{n}$ with coefficients in $\mathbb{C}$. A product of the form $x_{1}^{\alpha_{1}} x_{2}^{\alpha_{2}} \cdots x_{n}^{\alpha_{n}}$ is called a monomial in $x_{1}, x_{2}, \ldots, x_{n}$, where the $\alpha_{i}$ are nonnegative integers. The total degree of this monomial is the number $\sum_{i=1}^{n} \alpha_{i}$. A monomial is said to be square-free if all the $\alpha_{i}$ are at most 1 . It is obvious that an element $f$ of $\mathbb{C}\left[x_{1}, x_{2}, \ldots, x_{n}\right]$ can be uniquely written as a finite linear combination of monomials with coefficients in $\mathbb{C}$. The total degree of $f$ is the maximum total degrees of its monomials. We say that a polynomial $f$ is homogeneous of total degree $m$ if for all $t \in \mathbb{C}$,

$$
f\left(t x_{1}, t x_{2}, \ldots, t x_{n}\right)=t^{m} f\left(x_{1}, x_{2}, \ldots, x_{n}\right) .
$$

It can be easily seen that a polynomial is homogeneous if and only if all its monomials have the same total degrees.

The following, perhaps standard, theorem, which we have not found a reference for it, gives some information about the factors of a homogeneous polynomial. We give a proof of it for the convenience of the reader.

Theorem 2.1. Let $f, g, h \in \mathbb{C}\left[x_{1}, x_{2}, \ldots, x_{n}\right]$ be nonzero and $f=g h$. If $f$ is homogeneous, then $g$ and $h$ are also homogeneous.

Proof. Let $m, r$, and $s$ be the total degrees of $f, g$, and $h$, respectively. Then we may write $g$ and $h$ as follows:

$$
g=g_{r}+\cdots+g_{0}, \quad h=h_{s}+\cdots+h_{0},
$$

where $g_{k}$ and $h_{k}$ are the homogeneous parts of $g$ and $h$ of total degree $k$, respectively. Now let $i$ and $j$ be the least integers such that $g_{i} \neq 0 \neq h_{j}$. Therefore,

$$
f=g h=g_{i} h_{j}+l,
$$

where either $l=0$ or else $l$ is a polynomial with monomials of total degrees at least $i+j+1$. Thus $g_{i} h_{j} \neq 0$ is the homogeneous part of $f$ of total degree $i+j$. But $f$ is homogeneous of total degree $m$ and so it has a unique homogeneous part. We conclude that $l=0, i+j=m, i=r, j=s$. Thus $f=g_{r} h_{s}$ and the proof is complete. 
How the factors of a polynomial with square-free monomials can be is the statement of the next theorem.

Theorem 2.2. Let $f, g, h \in \mathbb{C}\left[x_{1}, x_{2}, \ldots, x_{n}\right]$ be nonzero and $f=g h$. If all monomials of $f$ are square-free, then all monomials of $g$ and $h$ are also squarefree and $g \in \mathbb{C}[A]$ and $h \in \mathbb{C}[B]$, where $\{A, B\}$ is a partition of $\left\{x_{1}, x_{2}, \ldots, x_{n}\right\}$.

Proof. By symmetry it suffices to show that all monomials of $g$ are squarefree and every indeterminate appearing in $g$ does not appear in $h$. Let $y \in$ $\left\{x_{1}, x_{2}, \ldots, x_{n}\right\}$ be an indeterminate which appears in $g$. Then $g$ and $h$ can be written as follows:

$$
g=a_{r} y^{r}+\cdots+a_{1} y+a_{0}, \quad h=b_{s} y^{s}+\cdots+b_{1} y+b_{0},
$$

where the $a_{i}$ and $b_{i}$ are polynomials in which the indeterminate $y$ does not appear, $a_{r} \neq 0 \neq b_{s}$, and $r \geq 1$ because $y$ appears in $g$. Therefore,

$$
f=g h=a_{r} b_{s} y^{r+s}+\cdots+\left(a_{1} b_{0}+a_{0} b_{1}\right) y+a_{0} b_{0} .
$$

But all monomials of $f$ are square-free, and so $r+s=1$. This implies that $r=1$ and $s=0$, completing the proof of the theorem.

Remark 1 . It is easy to see that if all the indeterminates $x_{1}, x_{2}, \ldots, x_{n}$ appear in $f$, then there is a unique partition $\{A, B\}$ of $\left\{x_{1}, x_{2}, \ldots, x_{n}\right\}$ such that $g \in \mathbb{C}[A]$ and $h \in \mathbb{C}[B]$.

It is known that the polynomial ring $\mathbb{C}\left[x_{1}, x_{2}, \ldots, x_{n}\right]$ is a UFD, that is, every nonconstant polynomial can be uniquely factored as a product of irreducible polynomials. Therefore, as a consequence we obtain the following:

Corollary 2.3. Let $f \in \mathbb{C}\left[x_{1}, x_{2}, \ldots, x_{n}\right]$ be a nonconstant polynomial with square-free monomials and $f_{1}, f_{2}, \ldots, f_{m}$ be the distinct irreducible factors of $f$. Then all monomials of the $f_{i}$ are also square-free, $f=f_{1} f_{2} \cdots f_{m}$, and $f_{i} \in \mathbb{C}\left[A_{i}\right]$, where $\left\{A_{1}, \ldots, A_{m}\right\}$ is a partition of $\left\{x_{1}, x_{2}, \ldots, x_{n}\right\}$.

Remark 2. It is obvious that $m \leq \operatorname{deg} f$. Also, the last part of the above corollary shows that $m \leq n$, and the equality holds if and only if, after reordering if necessary, $f_{i}=a_{i} x_{i}+b_{i}$ for some $a_{i}, b_{i} \in \mathbb{C}$.

Now we summarize some properties of the polynomial $d_{\chi}^{G}(X)$ in the ring $\mathbb{C}\left[x_{11}, x_{12}, \ldots, x_{n n}\right]$ in the next corollary.

Corollary 2.4. Let $G \leq S_{n}$, and $\chi: G \rightarrow \mathbb{C}$ be a nonzero function. Also let $f_{1}, f_{2}, \ldots, f_{m}$ be the distinct irreducible factors of $d_{\chi}^{G}(X)$. Then

i) $d_{\chi}^{G}(X)$ is a homogeneous polynomial of total degree $n$ with square-free monomials;

ii) the $f_{i}$ are homogeneous polynomials with square-free monomials, $m \leq$ $n$, and $d_{\chi}^{G}(X)=f_{1} f_{2} \cdots f_{m}$;

iii) $f_{i} \in \mathbb{C}\left[A_{i}\right]$, where $\left\{A_{1}, \ldots, A_{m}\right\}$ is a partition of $\left\{x_{11}, x_{12}, \ldots, x_{n n}\right\}$. 
For a given $f \in \mathbb{C}\left[x_{11}, x_{12}, \ldots, x_{n n}\right]$, let $B$ be the set of all indeterminates appearing in $f, R=\left\{r \mid \exists s, x_{r s} \in B\right\}$, and $C=\left\{s \mid \exists r, x_{r s} \in B\right\}$. In the next theorem we give detailed information about $d_{\chi}^{G}(X)$.

Theorem 2.5. Assume that the hypotheses of Corollary 2.4 hold. Let also $B_{i}$, $R_{i}$, and $C_{i}$ be as above for $f_{i}$. Then

i) if $i \neq j, x_{r s} \in B_{i}, x_{p q} \in B_{j}$, then $r \neq p, s \neq q$;

ii) if a monomial of $f_{i}$ contains distinct $x_{r s}, x_{p q}$, then $r \neq p, s \neq q$;

iii) $\left|R_{i}\right|=\left|C_{i}\right|=\operatorname{deg} f_{i}$

iv) the number of monomials of $f_{i}$ is at most $\left(\operatorname{deg} f_{i}\right)$ !;

$\mathrm{v})$ the number of monomials of $d_{\chi}^{G}(X)$ is at most $\prod_{i=1}^{m}\left(\operatorname{deg} f_{i}\right)$ !.

Proof. To prove (i), we may assume by way of contradiction that there exist $x_{r s} \in B_{i}$ and $x_{r q} \in B_{j}$ with $i \neq j$. By part (iii) of the previous corollary, we know that $x_{r s} \in A_{i}$ and $x_{r q} \in A_{j}$ and $A_{i}$ is disjoint from $A_{j}$. Hence there must exist a square-free monomial, say $g$, in $f_{1} f_{2} \cdots f_{m}$ containing the indeterminates $x_{r s}$ and $x_{r q}$. Since $d_{\chi}^{G}(X)=f_{1} f_{2} \cdots f_{m}$, thus there must be a $\sigma \in G$ such that $g=\prod_{i=1}^{n} x_{i \sigma(i)}$. This implies that $s=\sigma(r)=q$, saying that $A_{i}$ is not disjoint from $A_{j}$, a contradiction. Similarly, if there are $x_{r s} \in B_{i}$ and $x_{p s} \in B_{j}$ with $i \neq j$, then we can get a contradiction.

The same method as above can be used to prove (ii).

To obtain (iii), first we claim that if $\chi(\sigma) \neq 0$ for some $\sigma \in G$, then $\sigma\left(R_{i}\right) \subseteq$ $C_{i}$. Let $r \in R_{i}$ be arbitrary and so $x_{r s} \in B_{i}$ for some $s$. Since $\chi(\sigma) \neq 0$, hence the monomial $\prod_{i=1}^{n} x_{i \sigma(i)}$ must appear in $d_{\chi}^{G}(X)=f_{1} f_{2} \cdots f_{m}$. This implies that $x_{r \sigma(r)} \in B_{j}$ for some $j$. By part (i), $i=j$ and therefore $\sigma(r) \in C_{i}$. Now from $\chi$ being nonzero, we conclude that $\left\{R_{i}: 1 \leq i \leq m\right\}$ and $\left\{C_{i}: 1 \leq i \leq m\right\}$ are partitions of $\{1,2, \ldots, n\}$. Therefore by the claim we deduce that $\sigma\left(R_{i}\right)=$ $C_{i}$ for each $i$. Hence $\left|R_{i}\right|=\left|C_{i}\right|$. By part (ii), $\operatorname{deg} f_{i} \leq\left|R_{i}\right|$. But

$$
n=\sum_{i=1}^{m} \operatorname{deg} f_{i} \leq \sum_{i=1}^{m}\left|R_{i}\right|=n,
$$

and so $\operatorname{deg} f_{i}=\left|R_{i}\right|$ for each $i$.

By combining together parts (ii) and (iii), we obtain (iv).

Finally part (iv) implies (v).

Remark 3. The first part of the above theorem says that any two indeterminates appearing in two distinct $f_{i}$ do not lie in the same row or in the same column of the matrix $X=\left(x_{i j}\right)$. Also the second part says that no two indeterminates of $B_{i}$ which lie in the same row or in the same column of the matrix $X=\left(x_{i j}\right)$ can appear in the same monomial of $f_{i}$.

As a consequence we obtain the following:

Corollary 2.6. Let $G \leq S_{n}$, and $\chi: G \rightarrow \mathbb{C}$ be a nonzero function. Then

i) $m=n$ if and only if $d_{\chi}^{G}(X)$ is a multiple of a monomial if and only if there is a unique $\sigma \in G$ such that $\chi(\sigma) \neq 0$; 
ii) if $G=S_{n}$ and $\chi$ is nonzero everywhere, then $d_{\chi}^{G}(X)$ is irreducible. In particular, $\operatorname{det}(X)$ and $\operatorname{per}(X)$ are irreducible.

Proof. Part (i) can be deduced from part (iv) of the above theorem.

For part (ii), by hypothesis and part (v) of the above theorem we have

$$
\left(\sum_{i=1}^{m} \operatorname{deg} f_{i}\right) !=n !=\left|\left\{\sigma \in S_{n}: \chi(\sigma) \neq 0\right\}\right| \leq \prod_{i=1}^{m}\left(\operatorname{deg} f_{i}\right) ! \leq\left(\sum_{i=1}^{m} \operatorname{deg} f_{i}\right) !,
$$

hence the equality holds in the above. But this is possible if and only if $m=1$. This means that $d_{\chi}^{G}(X)$ must be irreducible.

Our next goal is to give a refinement of part (ii) of the above corollary.

Theorem 2.7. Let $G$ be the alternating group $A_{n}$ for $n \geq 4$ or the symmetric group $S_{n}$ for $n \geq 3$. Let also $\chi: G \rightarrow \mathbb{C}$ be a function which is nonzero on every element of some nontrivial conjugacy class of $G$. Then $d_{\chi}^{G}(X)$ is irreducible.

Proof. Suppose that $1 \neq \sigma \in G$ and $K$ is the conjugacy class of $\sigma$ in $G$ such that $\chi$ is nonzero on every element of $K$. Suppose also that $\sigma=\sigma_{1} \cdots \sigma_{r}$ is the decomposition of $\sigma$ into the nontrivial disjoint cycles and $\sigma_{1}=\left(i_{1} i_{2} \cdots i_{s}\right)$. Let $\left\{i_{s+1}, \ldots, i_{n}\right\}$ be the complement of $\left\{i_{1}, \ldots, i_{s}\right\}$ in $\{1,2, \ldots, n\}$. First assume that $n \geq 4$. Since $\chi(\sigma) \neq 0$, so by notation of Theorem 2.5 the monomial $\prod_{i=1}^{n} x_{i \sigma(i)}$, containing the indeterminate $x_{i_{1} i_{2}}$, must appear in $d_{\chi}^{G}(X)=$ $f_{1} f_{2} \cdots f_{m}$. Therefore, without loss of generality, we may assume that $x_{i_{1} i_{2}}$ appears in $f_{1}$ and hence $x_{i_{1} i_{2}} \in B_{1}$. Now let $i_{k} \neq i_{l}$ be arbitrary such that $\left\{i_{1}, i_{2}\right\} \cap\left\{i_{k}, i_{l}\right\}=\emptyset$. Consider the elements $\tau=\left(i_{2} i_{k} i_{l}\right), \nu=\left(i_{1} i_{2} i_{l}\right)$ and $\mu=\left(i_{1} i_{2}\right)\left(i_{k} i_{l}\right)$ from $G$. Now $\tau^{-1} \sigma \tau, \mu^{-1} \sigma \mu, \nu^{-1} \sigma \nu \in K$ and by hypothesis neither of $\chi\left(\tau^{-1} \sigma \tau\right), \chi\left(\nu^{-1} \sigma \nu\right), \chi\left(\mu^{-1} \sigma \mu\right)$ is zero. But $\left(\tau^{-1} \sigma \tau\right)\left(i_{1}\right)=i_{k}$, $\left(\nu^{-1} \sigma \nu\right)\left(i_{2}\right)=i_{l},\left(\mu^{-1} \sigma \mu\right)\left(i_{2}\right)=i_{1}$, and so in a similar manner as above, we deduce that $x_{i_{1} i_{k}}, x_{i_{2} i_{l}}, x_{i_{2} i_{1}}$ all must appear in $d_{\chi}^{G}(X)$. Since $x_{i_{1} i_{2}} \in B_{1}$, hence by part (i) of Theorem 2.5, we have $x_{i_{1} i_{k}} \in B_{1}$, which in turn implies that $\left\{i_{2}, \ldots, i_{n}\right\} \subseteq C_{1}$. In particular, $x_{i_{1} i_{l}} \in B_{1}$, and again by part (i) of Theorem 2.5 we have $x_{i_{2} i_{l}} \in B_{1}$. Finally by the same reason $x_{i_{2} i_{1}} \in B_{1}$. This means that $i_{1} \in C_{1}$ and so $\operatorname{deg} f_{1}=\left|C_{1}\right|=n$, showing that $d_{\chi}^{G}(X)=f_{1}$ and the proof is complete in this case. The assertion can be proved similarly when $G=S_{3}$.

Remark 4. The above theorem may be false if $G$ is different from $S_{n}$ or $A_{n}$. For example, if we let $G=\{1,(12),(34),(12)(34)\} \leq S_{4}$ and $\chi=1_{G}$ be the principal character of $G$, then $d_{\chi}^{G}(X)$ is reducible since

$$
d_{\chi}^{G}(X)=\left(x_{11} x_{22}+x_{12} x_{21}\right)\left(x_{33} x_{44}+x_{34} x_{43}\right) .
$$

The following result is a consequence of the above theorem.

Corollary 2.8. Let $G=A_{n}$ for $n \neq 2$ or $G=S_{n}$ and $\chi$ be an irreducible character of $G$. Then $d_{\chi}^{G}(X)$ is irreducible. 


\section{On $\chi$-singular and $\chi$-nonsingular matrices}

From linear algebra we know that a matrix $A \in M_{n}(\mathbb{C})$ is called singular if $\operatorname{det}(A)=0$ and nonsingular if $\operatorname{det}(A) \neq 0$, that is, $d_{\varepsilon}^{S_{n}}(A)=0$ and $d_{\varepsilon}^{S_{n}}(A) \neq 0$, respectively. Motivated by this, we introduce a more general concept. Let $G \leq S_{n}$ and $\chi: G \rightarrow \mathbb{C}$ be a function. A matrix $A \in M_{n}(\mathbb{C})$ is said to be $\chi$ singular if $d_{\chi}^{G}(A)=0$ and $\chi$-nonsingular if $d_{\chi}^{G}(A) \neq 0$. So an ordinary singular (nonsingular) matrix is in fact an $\varepsilon$-singular ( $\varepsilon$-nonsingular) matrix. Also a matrix $A \in M_{n}(\mathbb{C})$ is $1_{S_{n}}$-singular if and only if $\operatorname{per}(A)=d_{1_{S_{n}}}^{S_{n}}(A)=0$. The following is Theorem 2.1 in [3].

Theorem 3.1. Let $G \leq S_{n}$ and $\chi: G \rightarrow \mathbb{C}$ be a nonzero function. Then the following are equivalent:

i) $d_{\chi}^{G}(A) \neq 0$ for all $\varepsilon$-nonsingular matrices $A$;

ii) $d_{\chi}^{G}(A)=0$ for all $\varepsilon$-singular matrices $A$;

iii) $G=S_{n}$ and $\chi=\chi(1) \varepsilon$.

The above theorem as well as all other results of [3] with slight changes in proofs remain true if one substitutes $1_{S_{n}}$ for $\varepsilon$. Our next goal is to generalize the above theorem. Unfortunately, the proof given in [3] does not work in the general case and so we have to take a totally different approach.

Before stating the next theorem, we recall a few notation, definitions, and Hilbert's Nullstellensatz from [2].

Let $R$ be a commutative ring with identity and $I$ an ideal of $R$. The radical of $I$, denoted by $\sqrt{I}$ or $\operatorname{rad}(I)$, is defined as follows:

$$
\sqrt{I}=\left\{r \in R \mid r^{n} \in I \text { for some } n \in \mathbb{N}\right\},
$$

which is obviously an ideal of $R$ containing $I$.

For a subset $T$ of $\mathbb{C}^{n}$ we define $\mathcal{I}(T)$ as follows:

$\mathcal{I}(T)=\left\{f \in \mathbb{C}\left[x_{1}, x_{2}, \ldots, x_{n}\right] \mid f\left(a_{1}, a_{2}, \ldots, a_{n}\right)=0\right.$ for all $\left.\left(a_{1}, a_{2}, \ldots, a_{n}\right) \in T\right\}$, which is certainly an ideal of $\mathbb{C}\left[x_{1}, x_{2}, \ldots, x_{n}\right]$.

Also for a subset $S$ of $\mathbb{C}\left[x_{1}, x_{2}, \ldots, x_{n}\right]$ we define $\mathcal{Z}(S)$ as follows:

$$
\mathcal{Z}(S)=\left\{\left(a_{1}, a_{2}, \ldots, a_{n}\right) \in \mathbb{C}^{n} \mid f\left(a_{1}, a_{2}, \ldots, a_{n}\right)=0 \text { for all } f \in S\right\} .
$$

Now Hilbert's Nullstellensatz states that if $I$ is an ideal of $\mathbb{C}\left[x_{1}, x_{2}, \ldots, x_{n}\right]$, then $\mathcal{I}(\mathcal{Z}(I))=\sqrt{I}$.

We are now ready to prove the next theorem from which our generalization of the above theorem will be derived. Though the following theorem has been proved in [1], but our proof, which uses Hilbert's Nullstellensatz and results of the previous section, is entirely different than theirs.

Theorem 3.2. Let $\chi, \varphi: S_{n} \rightarrow \mathbb{C}$ be two functions. If every $\chi$-singular matrix of $M_{n}(\mathbb{C})$ is a $\varphi$-singular matrix, then there is a $c \in \mathbb{C}$ such that $\varphi=c \chi$. 
Proof. If $\chi=0$, then all matrices in $M_{n}(\mathbb{C})$ are $\chi$-singular and so, by hypothesis, are $\varphi$-singular. Hence for an arbitrary $\sigma \in S_{n}$, we have $\varphi(\sigma)=d_{\varphi}^{S_{n}}\left(A_{\sigma}\right)=0$, where $A_{\sigma}$ is the permutation matrix induced by $\sigma$. Thus $\varphi=0$ and the result follows in this case. Also, if $\varphi=0$, then by taking $c=0$ the result follows too.

Assume now that $\chi \neq 0 \neq \varphi$. Therefore $d_{\chi}^{S_{n}}(X)$ and $d_{\varphi}^{S_{n}}(X)$ are nonzero homogeneous polynomials with the same total degree $n$. Let $I$ and $J$ be the ideals of the ring $\mathbb{C}\left[x_{11}, x_{12}, \ldots, x_{n n}\right]$ generated by the polynomials $d_{\chi}^{S_{n}}(X)$ and $d_{\varphi}^{S_{n}}(X)$, respectively. By hypothesis we have $\mathcal{Z}(I) \subseteq \mathcal{Z}(J)$ and hence $\mathcal{I}(\mathcal{Z}(J)) \subseteq \mathcal{I}(\mathcal{Z}(I))$. Therefore $\sqrt{J} \subseteq \sqrt{I}$ by Hilbert's Nullstellensatz, and since $d_{\varphi}^{S_{n}}(X) \in J \subseteq \sqrt{J}$ we obtain $\left(d_{\varphi}^{S_{n}}(X)\right)^{k} \in I$ for some $k \in \mathbb{N}$. This implies that $\left(d_{\varphi}^{S_{n}}(X)\right)^{k}$ is divisible by $d_{\chi}^{S_{n}}(X)$. Therefore, $d_{\varphi}^{S_{n}}(X)$ is divisible by $d_{\chi}^{S_{n}}(X)$ by part (ii) of Corollary 2.4. So there exists a $c \in \mathbb{C}$ such that $d_{\varphi}^{S_{n}}(X)=c d_{\chi}^{S_{n}}(X)$. This means that $\varphi=c \chi$ and the proof is complete.

Before giving the next results, we introduce a notation. For $G \leq S_{n}$ and $\chi$ a complex valued function defined on $G$, let $\hat{\chi}$ be an extension of $\chi$ to $S_{n}$ which vanishes outside of $G$. It is obvious that $d_{\chi}^{G}=d_{\hat{\chi}}^{S_{n}}$. Hence a matrix $A \in M_{n}(\mathbb{C})$ is $\chi$-singular if and only if it is $\hat{\chi}^{\text {-singular. The next consequence }}$ is a generalization of Theorem 3.1 .

Corollary 3.3. Let $H$ and $K$ be two subgroups of $S_{n}$, and let $\chi: H \rightarrow \mathbb{C}$ and $\varphi: K \rightarrow \mathbb{C}$ be two nonzero functions. Then the following are equivalent:

i) every $\varphi$-nonsingular matrix of $M_{n}(\mathbb{C})$ is a $\chi$-nonsingular matrix;

ii) every $\chi$-singular matrix of $M_{n}(\mathbb{C})$ is a $\varphi$-singular matrix;

iii) $\chi$ and $\varphi$ vanish outside of $H \cap K$, and there exists a $c \in \mathbb{C}$ such that $\varphi_{H \cap K}=c \chi_{H \cap K}$

iv) $\chi$ and $\varphi$ vanish outside of $H \cap K$, and there exists a $c \in \mathbb{C}$ such that $\chi_{H \cap K}=c \varphi_{H \cap K}$

v) every $\varphi$-singular matrix of $M_{n}(\mathbb{C})$ is a $\chi$-singular matrix;

vi) every $\chi$-nonsingular matrix of $M_{n}(\mathbb{C})$ is a $\varphi$-nonsingular matrix.

Proof. Let $\hat{\chi}$ and $\hat{\varphi}$ be as above. Since a matrix $A \in M_{n}(\mathbb{C})$ is $\chi$-singular ( $\varphi$-singular) if and only if it is $\hat{\chi}$-singular $(\hat{\varphi}$-singular $)$, the result follows from Theorem 3.2.

The following theorem can be viewed as a generalization of Theorem 2.2 of [3].

Theorem 3.4. Let $G, H, K \leq S_{n}$, and $\chi, \varphi, \psi$ be complex valued functions defined on $G, H, K$, respectively, with $\chi(1) \neq 0$. Then the following are equivalent:

i) $d_{\varphi}^{H}(A)=d_{\psi}^{K}(A)$ for all $\chi$-nonsingular matrices $A$;

ii) $d_{\varphi}^{H}(A)=d_{\psi}^{K}(A)$ for all $\chi$-singular matrices $A$, and $\varphi(1)=\psi(1)$;

iii) $\varphi$ and $\psi$ vanish outside of $H \cap K$, and $\varphi_{H \cap K}=\psi_{H \cap K}$.

Proof. It is trivial that (iii) implies (i) and (ii). 
We now show that (i) implies (iii). It is sufficient to show the claim $\hat{\varphi}(\sigma)=$ $\hat{\psi}(\sigma)$ for all $\sigma \in S_{n}$. Let $\sigma=\sigma_{1} \cdots \sigma_{r}$ be the decomposition of $\sigma$ into the nontrivial disjoint cycles. We prove the above claim by induction on $r$. If $r=0$ then $\sigma=1$, and since $I_{n}$ is $\hat{\chi}$-nonsingular by hypothesis, so

$$
\hat{\varphi}(1)=d_{\hat{\varphi}}^{S_{n}}\left(I_{n}\right)=d_{\hat{\psi}}^{S_{n}}\left(I_{n}\right)=\hat{\psi}(1) .
$$

Assume now that $r>0$. If $\hat{\chi}(\sigma) \neq 0$, then $A_{\sigma}$, the permutation matrix induced by $\sigma$, is $\hat{\chi}$-nonsingular and so

$$
\hat{\varphi}(\sigma)=d_{\hat{\varphi}}^{S_{n}}\left(A_{\sigma}\right)=d_{\hat{\psi}}^{S_{n}}\left(A_{\sigma}\right)=\hat{\psi}(\sigma) .
$$

Therefore we may assume that $\hat{\chi}(\sigma)=0$. Since $\chi(1) \neq 0$, so there exists a permutation $\tau$ so that it can be written as a product of $s$ distinct elements of the set $\left\{\sigma_{1}, \ldots, \sigma_{r}\right\}$ with $\hat{\chi}(\tau) \neq 0$ and $s$ as maximum as possible. Obviously $0 \leq s<r$. Without loss of generality, we may assume that $\tau=\sigma_{1} \cdots \sigma_{s}$. Let $\Gamma_{0}=\{1\}$ and for $1 \leq k \leq r-s$, let $\Gamma_{k}$ be the set of all permutations that can be written as a product of $k$ distinct elements of the set $\left\{\sigma_{s+1}, \ldots, \sigma_{r}\right\}$. We define the diagonal matrix $B=\left(b_{i j}\right) \in M_{n}(\mathbb{C})$ as follows:

$$
b_{i j}=\left\{\begin{array}{cl}
\delta_{i j} & i \in \operatorname{Fix}(\tau)-\operatorname{Fix}(\sigma) \\
0 & i \notin \operatorname{Fix}(\tau)-\operatorname{Fix}(\sigma) .
\end{array}\right.
$$

If $A=A_{\sigma}+B$, then by the maximality of $s$ we have

$$
d_{\hat{\chi}}^{S_{n}}(A)=\sum_{k=0}^{r-s} \sum_{\lambda \in \Gamma_{k}} \hat{\chi}(\tau \lambda)=\hat{\chi}(\tau) \neq 0 .
$$

This implies that the matrix $A$ is $\hat{\chi}$-nonsingular and so by hypothesis we obtain

$$
\hat{\varphi}(\sigma)+\sum_{k=0}^{r-s-1} \sum_{\lambda \in \Gamma_{k}} \hat{\varphi}(\tau \lambda)=d_{\hat{\varphi}}^{S_{n}}(A)=d_{\hat{\psi}}^{S_{n}}(A)=\hat{\psi}(\sigma)+\sum_{k=0}^{r-s-1} \sum_{\lambda \in \Gamma_{k}} \hat{\psi}(\tau \lambda) .
$$

But by induction we have

$$
\sum_{k=0}^{r-s-1} \sum_{\lambda \in \Gamma_{k}} \hat{\varphi}(\tau \lambda)=\sum_{k=0}^{r-s-1} \sum_{\lambda \in \Gamma_{k}} \hat{\psi}(\tau \lambda) .
$$

Therefore $\hat{\varphi}(\sigma)=\hat{\psi}(\sigma)$, and the result follows.

Finally we show that (ii) implies (iii). By hypothesis, for all $\hat{\chi}$-singular matrices $A$, we have

$$
d_{\hat{\varphi}}^{S_{n}}(A)=d_{\varphi}^{H}(A)=d_{\psi}^{K}(A)=d_{\hat{\psi}}^{S_{n}}(A),
$$

and so $d_{\hat{\varphi}-\hat{\psi}}^{S_{n}}(A)=0$. Thus by Theorem $3.2, \hat{\varphi}-\hat{\psi}=c \hat{\chi}$ for some $c \in \mathbb{C}$. Since $\chi(1) \neq 0$ and $\varphi(1)=\psi(1)$, so $c=0$. This implies that $\hat{\varphi}=\hat{\psi}$, and the proof is complete. 
Remark 5. First note that if $\chi$ is a character of $G$, then automatically $\chi(1) \neq 0$. Also, the condition $\varphi(1)=\psi(1)$ in case (ii) of the above theorem is essential. This is because if $\varphi$ is any function of $S_{n}$, then $d_{\varphi}^{S_{n}}(A)=d_{\varphi+\varepsilon}^{S_{n}}(A)$ for all $\varepsilon$ singular matrices $A$. Of course, the authors in Theorem 2.2 of [3] showed that the condition $\varphi(1)=\psi(1)$ can be removed if $G=S_{n}, \chi=\varepsilon$ or $1_{S_{n}}$, and $\varphi$ and $\psi$ are irreducible characters. But, in general, we cannot expect to get a similar theorem here. For example, let $G=H=A_{3}$ and $K=S_{3}$. Also let $\chi, \varphi$ be the two distinct nonprincipal linear characters of $A_{3}$ and $\psi$ be the nonlinear irreducible character of $S_{3}$. It is obvious that $\psi=\hat{\chi}+\hat{\varphi}$ and therefore for all $\chi$-singular matrices $A$ we have

$$
d_{\psi}^{K}(A)=d_{\hat{\chi}+\hat{\varphi}}^{S_{3}}(A)=d_{\hat{\chi}}^{S_{3}}(A)+d_{\hat{\varphi}}^{S_{3}}(A)=d_{\varphi}^{H}(A) .
$$

\title{
References
}

[1] L. B. Beasley and L. J. Cummings, On the uniqueness of generalized matrix functions, Proc. Amer. Math. Soc. 87 (1983), no. 2, 229-232.

[2] D. S. Dummit and R. M. Foote, Abstract Algebra, John Wiley and Sons, Inc., 2004.

[3] M. H. Jafari and A. R. Madadi, On the equality of generalized matrix functions, Linear Algebra Appl. 456 (2014), 16-21.

[4] M. Marcus, Finite Dimensional Multilinear Algebra, Part I, Marcel Dekker, 1973.

[5] R. Merris, Multilinear Algebra, Gordon and Breach Science Publishers, 1997.

\author{
Mohammad Hossein Jafari \\ Department of Pure Mathematics \\ Faculty of Mathematical Sciences \\ UNIVERSITY OF TABRIZ \\ TABRIZ, IRAN \\ E-mail address: jafari@tabrizu.ac.ir
}

Ali Reza Madadi

Department of Pure Mathematics

Faculty of Mathematical Sciences

UNIVERSITY OF TABRIZ

TABRIZ, IRAN

E-mail address: a-madadi@tabrizu.ac.ir 